\title{
THE RICCI FLOW ON NONCOMMUTATIVE TWO-TORI
}

\author{
TANVIR AHAMED BHUYAIN AND MATILDE MARCOLLI
}

\begin{abstract}
In this paper we construct a version of Ricci flow for noncommutative 2-tori, based on a spectral formulation in terms of the eigenvalues and eigenfunction of the Laplacian and recent results on the Gauss-Bonnet theorem for noncommutative tori.
\end{abstract}

\section{INTRODUCTION}

The purpose of this paper is to identify and describe an appropriate analog of Hamilton's Ricci flow for the noncommutative two tori, which are the prototype example of noncommutative manifolds. Our main result is that we can express the Ricci flow as an equation

$$
\frac{d \lambda}{d t}=\lambda 12 \pi \zeta_{f^{2}}(0)
$$

for the eigenvalues and the corresponding eigenfunctions of the Laplacian $\triangle^{\prime} \sim$ $k \triangle k$ considered in [20, with the zeta function $\zeta_{a}(s)=\operatorname{Tr}\left(a \triangle^{-s}\right)$. Moreover, (1.1) admits a reformulation in terms of pseudodifferential calculus and the properties of the modular operator of the associated non-unimodular spectral geometry on the noncommutative torus, as in 20. This gives then an equivalent formulation of (1.1) as

$$
\frac{d \lambda}{d t}=\lambda \tau\left(f^{2} \mathcal{R}\right)
$$

where $\tau$ is the unique normalized trace (2.1) on the noncommutative torus and $\mathcal{R}$ is obtained as

$$
\frac{-1}{12 \pi^{2}} \mathcal{R}=2 \int_{0}^{\infty} T(r) b_{0} r d r
$$

where, as in [20], one has $\sigma\left(B_{u}\right)(\xi)=b_{0}(\xi)+b_{1}(\xi)+b_{2}(\xi)+\cdots$ the symbol of $B_{u}=\left(u \mathbf{1}-\triangle^{\prime}\right)^{-1}$, and $T(r)$ has an explicit expression (3.7) involving the symbol. This can then be re-expressed in terms of the modular operator $\Delta(a)=e^{-h} a e^{h}$ with $a \in \mathcal{A}_{\theta}^{\infty}$ of the non-unimodular geometry $k \triangle k$ with $k=e^{h / 2}$, as shown in Theorem 3.5 .

1.1. The Ricci flow and noncommutative geometry. Hamilton's Ricci flow [32] on Riemannian manifolds $(M, g)$ is given by the evolution equation

$$
\frac{\partial g}{\partial t}=-2 \operatorname{Ric}(g) \text {. }
$$

The Ricci flow became a fundamental tool in the study of the geometry and topology of manifolds, starting with the seminal results of Hamilton [32, [35], 36], [37, and culminating more recently with Perelman's proof of the Poincaré conjecture 49, [50, [51, see also [1, 47] and [10, [4], 44]. 
The Ricci flow also found many interesting applications in physics: for instance in the renormalization group equations of 2-dimensional sigma models [2], in the evolution of the ADM mass in asymptotically flat spaces [24, and also, recently, in studying the contribution of black holes in Euclidean quantum gravity [31. In view of both the mathematical and the physical interest, and of the emergence of noncommutative spaces as both an extension of Riemannian geometry [17, [16] and as a proposed setting for gravity models, we consider it to be an interesting problem to derive extensions of the Ricci flow in the setting of noncommutative geometry.

A possible extension of the Ricci flow to noncommutative spaces was proposed in [54], within the spectral action paradigm for noncommutative geometry (see [12, 17]), according to which the analog of a (spin) Riemannian manifold in noncommutative geometry is described by a spectral triple $(\mathcal{A}, \mathcal{H}, D)$ as in [17, with $\mathcal{A}$ a (noncommutative) algebra of "smooth functions", $\mathcal{H}$ a Hilbert space representation (the noncommutative analog of square integrable spinors) and $D$ a Dirac operator. Euclidean gravity on such a noncommutative manifold is then described by the spectral action functional of [12, which can be thought of as a modified gravity model. The approach developed in 54 aims at defining an analog of Ricci flow on noncommutative spaces by working directly in terms of the spectral action functional.

The approach we follow in this paper is different from the one proposed in [54] and it relies on generalizing to the noncommutative setting the behavior of Laplace eigenvalues under the Ricci flow on ordinary manifolds, seen as providing a suitable spectral version of the Ricci flow.

1.2. The Ricci flow and the Laplace spectrum. The behavior of eigenvalues of the Laplacian under the Ricci flow on Riemannian manifolds is a subtle and important question: it is related, for instance, to Perelman's monotonicity and entropy result in 49 , which shows that the lowest eigenvalue of the operator $-4 \triangle+$ $R$ is non-decreasing along the Ricci flow, and to other results of a similar flavor in [8, [9, 25], 41, 43.

Here, in order to identify a prototype commutative model of what will happen for noncommutative tori, we focus on the result of [25, where Luca Fabrizio Di Cerbo provides an equation for the evolution of Laplace eigenvalues, under the very strong assumption of the existence and $\mathcal{C}^{1}$-differentiability of the eigenvalues $\lambda_{i}(t)$ and eigenfunctions $f_{i}(t)$, along the deformation $g(t)$ of the initial metric determined by the Ricci flow. This strong assumption, which is in some sense an analog of a Berger and Bando-Urakawa deformation result [6, 4, may be too strong for arbitrary manifolds (see the comments in [25]). However, since we are interested in the particular case of the 2-dimensional noncommutative tori, we concentrate here only on the commutative case of 2-dimensional surfaces, and in particular of the commutative 2-torus.

The properties of the Ricci flow in 2-dimension were studied in [33] and [13, 34, 38. As shown in [33], in 2-dimension the Ricci flow is related to the gradient flow of the Yamabe problem. For any initial data, solutions exist for all time. For surfaces of genus $g \geq 1$, in was shown in [33] that any metric flows to a constant curvature metric and in the genus zero case any metric with positive Gauss curvature also flows to a constant curvature metric. The latter assumption in the genus zero case 
was removed in [13] by showing that, for any metric on $S^{2}$, the Gaussian curvature becomes positive in finite time under the Ricci flow. In particular, in the case of a commutative 2-dimensional torus, an arbitrary initial metric flows to the flat metric under the Ricci flow, 33.

In the 2-dimensional case, under the assumptions described above, the equation for the Laplace eigenvalues under the Ricci flow on a closed surface $M$ is given in Corollary 2.3 of $[25]$ as

$$
\frac{d \lambda}{d t}=\lambda \int_{M} f^{2} R d \mu
$$

where $f=f_{t}$ is the normalized evolving eigenfunction associated to the evolving eigenvalue $\lambda=\lambda(t)$, with

$$
\int_{M} f d \mu=0 \quad \text { and } \quad \int_{M} f^{2} d \mu=1 .
$$

1.3. The Ricci flow and noncommutative tori. Our key step in extending (1.3) to the noncommutative case lies in the formulation of the Gauss-Bonnet theorem for noncommutative tori obtained by Connes-Tretkoff in [20].

In the Gauss-Bonnet theorem, the analog of the curvature integral that computes the Euler characteristic is given in terms of the value at zero of the zeta function of the Laplacian on the noncommutative torus, and this, in turn, is computed explicitly in terms of pseudodifferential calculus on the noncommutative space, and proved to be independent of the Weyl factor as in the classical case.

In our approach to the Ricci flow, we adapt the same technique to deal with the values at zero of a more general class of zeta functions associated to the noncommutative geometry, those of the form $\zeta_{a}(s)=\operatorname{Tr}\left(a \Delta^{-s}\right)$.

We then conclude the paper by mentioning some possible applications of this noncommutative extension of the Ricci flow, both in the mathematical context and in terms of applications to physics.

\section{RiCCI FLOW AND ZETA FUNCTIONS}

In this section we derive the formula for the Ricci flow on a noncommutative torus in terms of zeta functions. We begin by recalling briefly the setting of [20] on noncommutative tori, variations of the metric within a conformal class, and the Gauss-Bonnet theorem.

2.1. Noncommutative tori. We recall some basic properties of noncommutative tori, which we will need later, following [15] and [20].

Let $\theta$ be an irrational number. Then the irrational rotation $C^{*}$-algebra $\mathcal{A}_{\theta}$ is the universal unital $C^{*}$-algebra generated by two unitaries $U, V$ which satisfy the relation

$$
U V=e^{2 \pi i \theta} V U, \quad \text { with } \quad U^{*} U=U U^{*}=1=V^{*} V=V V^{*} .
$$

The algebra $\mathcal{A}_{\theta}$ is thought of as continuous functions on the noncommutative twotorus $\mathbb{T}_{\theta}^{2}$, [15].

A continuous action of $\mathbb{T}^{2}$, with $(\mathbb{T}=\mathbb{R} / 2 \pi \mathbb{Z})$, on $\mathcal{A}_{\theta}$ is given by the twoparameter group of automorphism $\left\{\alpha_{s}\right\}$, for $\left(s \in \mathbb{R}^{2}\right)$, determined by

$$
\alpha_{s}\left(U^{n} V^{m}\right)=e^{i s .(n, m)} U^{n} V^{m} .
$$


The subalgebra of elements $a \in \mathcal{A}_{\theta}$ for which the map $s \mapsto \alpha_{s}(a)$ is smooth is denoted by $A_{\theta}^{\infty}$. Alternatively, as in [15] one has

$$
\mathcal{A}_{\theta}^{\infty}=\left\{\sum_{m, n \in \mathbb{Z}} a_{m, n} U^{m} V^{n}:\left(|m|^{k}|n|^{q}\left|a_{m, n}\right|\right) \text { is bounded for any positive } k, q\right\} .
$$

The corresponding derivations are given by

$$
\begin{aligned}
& \delta_{1}(U)=U, \delta_{1}(V)=0, \\
& \delta_{2}(U)=0, \delta_{2}(V)=V .
\end{aligned}
$$

These derivations are viewed as the analogs to the differential operators $-i \partial / \partial x$, $-i \partial / \partial y$ of the classical case. We also have

$$
\delta_{j}\left(a^{*}\right)=-\delta_{j}(a)^{*}, j=1,2 .
$$

For irrational $\theta$, there is a unique normalized trace $\tau$ on $\mathcal{A}_{\theta}$ given by

$$
\tau\left(U^{n} V^{m}\right)=0 \quad \text { if } \quad(n, m) \neq(0,0), \quad \text { and } \quad \tau(1)=1 .
$$

This satisfies the analog of the classical integration by parts formula

$$
\tau \circ \delta_{j}=0, \tau\left(a \delta_{j}(b)\right)=-\tau\left(\delta_{j}(a) b\right), \forall a, b \in \mathcal{A}_{\theta}^{\infty}, j=1,2 .
$$

More details on the structure of the noncommutative torus can be found, for example, in [16, [55].

It is customary to consider a Hilbert space $H_{0}$ constructed as the completion of $\mathcal{A}_{\theta}$ with respect to the inner product

$$
\langle a, b\rangle=\tau\left(b^{*} a\right), \quad a, b \in \mathcal{A}_{\theta} .
$$

With respect to this inner product, $\delta_{1}, \delta_{2}$ are formally self adjoint unbounded operators on $H_{0}$. One can then add a complex structure by defining

$$
\partial=\delta_{1}+i \delta_{2}, \quad \partial^{*}=\delta_{1}-i \delta_{2} .
$$

The operator $\partial$ is then an unbounded operator on $H_{0}$ and $\partial^{*}$ is its formal adjoint with respect to the inner product determined by $\tau$.

The information on the conformal structure is contained in the positive Hochschild two-cocycle described in [18] and [16,

$$
\psi(a, b, c)=-\tau\left(a \partial b \partial^{*} c\right), \quad a, b, c \in \mathcal{A}_{\theta}^{\infty} .
$$

The analog of the classical space of $(1,0)$-forms is the unitary bimodule $H^{(1,0)}$ on $\mathcal{A}_{\theta}$ obtained by the Hilbert space completion of the space of finite sums $\sum a \partial b$, where $a, b \in \mathcal{A}_{\theta}^{\infty}$, with respect to the inner product described above. We consider $\partial$ to be an unbounded operator from $H_{0}$ to $H^{(1,0)}$. The Laplacian $\triangle$ on the functions on $\mathbb{T}_{\theta}^{2}$ is then defined as

$$
\triangle=\partial^{*} \partial=\delta_{1}^{2}+\delta_{2}^{2} .
$$


2.2. Variation of the metric and the Laplacian. We continue with our brief review of the notions of [20] that we will need to use in the following.

We vary inside the conformal class of a metric by choosing a self adjoint element $h \in A_{\theta}^{\infty}$ and defining the positive linear functional

$$
\varphi(a)=\tau\left(a e^{-h}\right), \quad a \in \mathcal{A}_{\theta} .
$$

One constructs then a unitary left module $H_{\varphi}$ on $\mathcal{A}_{\theta}$ by completing $\mathcal{A}_{\theta}$ with respect to the inner product

$$
\langle a, b\rangle_{\varphi}=\varphi\left(b^{*} a\right), \quad a, b \in A_{\theta} .
$$

One then considers $\partial_{\varphi}$, which is the same operator as $\partial$ but viewed as an unbounded operator from $H_{\varphi}$ to $H^{(1,0)}$, and one constructs the modified Laplacian $\triangle^{\prime}$ as a positive unbounded operator acting in $H_{\varphi}$ given by

$$
\triangle^{\prime}=\partial_{\varphi}^{*} \partial_{\varphi}
$$

One then has the following result.

Lemma 2.1. (Connes-Tretkoff [20]) The operator $\triangle^{\prime}$ acting on $H_{\varphi}$ is anti-unitarily equivalent to the positive unbounded operator $k \triangle k$ acting on $H_{0}$, where $k=e^{h / 2} \in$ $\mathcal{A}_{\theta}$ acts by left multiplication on $H_{0}$.

We refer the reader to 20 for more details.

By Lemma 2.1, one sees that $\triangle^{\prime}$ and $k \triangle k$ have the same eigenvalues. Thus, we will be considering an analog of the Ricci flow equation (1.3) obtained by Di Cerbo in 25, in the form of a time evolution equation for the operator $k \triangle k$ involving its eigenvalues. This will give us an evolution of $k=e^{h / 2}$ and subsequently an evolution for $h \in A_{\theta}^{\infty}$, which parametrizes the metric $\varphi$ (through the inner product induced by $\varphi$ ), with $\varphi(a)=\tau\left(a e^{-h}\right)$ as above.

2.3. Zeta functions for noncommutative tori. For a classical (commutative) closed 2-dimensional surface (in particular the two torus), the spectral zeta function of the Laplacian $\triangle$ is given by

$$
\zeta(s)=\sum_{j} \lambda_{j}^{-s}=\operatorname{Tr}\left(\triangle^{-s}\right), \quad \text { for } \operatorname{Re}(s)>1,
$$

where $\lambda_{j}$ are the eigenvalues of $\triangle$ and we have assumed that $\operatorname{Ker}(\triangle)=0$.

The function $\zeta(s)$ has a meromorphic continuation to the origin $s=0$, where it has no pole, and we have (still assuming $\operatorname{Ker}(\triangle)=0$ )

$$
\zeta(0)=\frac{1}{12 \pi} \int_{M} R d \mu=\frac{1}{6} \chi(M),
$$

with $\chi(M)$ the topological Euler characteristic (by Gauss-Bonnet theorem), which vanishes in the case of the 2 -torus $\mathbb{T}^{2}$.

According to a general philosophy developed recently in [21, 222, 23], a good "set of coordinates" on a noncommutative geometry is provided by a family of zeta functions, which generalize the zeta function of the Laplacian $\triangle$ (or of a Dirac operator $D$ ) by including elements of the algebra $\mathcal{A}$, in the form

$$
\zeta_{a}(s)=\operatorname{Tr}\left(a \triangle^{-s}\right) .
$$

In the case of an ordinary Riemannian manifold, it is proved in 21] that a similar family of zeta functions reconstructs the manifold up to isometry. Here we will use 
specific zeta functions in this family to extend the integration (2.6) to an expression that recovers, for ordinary (commutative) 2-tori, the eigenvalue equation for the Ricci flow of [25] and continues to make sense for all the noncommutative 2-tori.

Proposition 2.2. In the case of the closed 2-dimensional surface $M$, let $f$ be an element in the smooth subalgebra $C^{\infty}(M)$ and consider the zeta function $\zeta_{f}(s)=$ $\operatorname{Tr}\left(f \triangle^{-s}\right)$ as above. Then $\zeta_{f}(s)$ has a meromorphic continuation at $s=0$ where it satisfies

$$
\zeta_{f}(0)=\frac{1}{12 \pi} \int_{M} f R d \mu .
$$

Proof. We can work under the assumptions of Lemma 1.2.1 of [30], see also (1.12.14) of [30, since $\operatorname{Tr}\left(f e^{-t \triangle}\right)$ is admissible in the sense specified there. Then $\zeta_{f}(s)=$ $\operatorname{Tr}\left(f \triangle^{-s}\right)$ can be written via Mellin transform as

$$
\operatorname{Tr}\left(f \triangle^{-s}\right)=\frac{1}{\Gamma(s)} \int_{0}^{\infty} \operatorname{Tr}\left(f e^{-t \triangle}\right) t^{s-1} d t .
$$

The function $\Gamma(s) \zeta_{f}(s)$ has a meromorphic extension to $\mathbb{C}$, with residues given in terms of the coefficients $a_{n}$ of the heat-kernel expansion, see Theorem 1.12.2 of 30. In particular, $\zeta_{f}(s)$ is regular at $s=0$. As in 30, we have in general for $m=\operatorname{dim} M$,

$$
\operatorname{Tr}\left(f e^{-t \triangle}\right) \sim \sum_{n} t^{(n-m) / 2} \int_{M} f a_{n}(\triangle) d \mu,
$$

so that, combining this with what we already know from (2.6), we obtain as the value $\zeta_{f}(0)$ the integration (2.8).

We can then reformulate the equation (1.3) in terms of the zeta functions as follows.

Corollary 2.3. Let $M$ be a closed 2-dimensional surface. The Ricci flow equation (1.3) for the eigenvalues of the Laplacian can be rewritten as

$$
\frac{d \lambda}{d t}=\lambda 12 \pi \zeta_{f^{2}}(0)
$$

where $\lambda$ is an evolving eigenvalue and $f$ the corresponding normalized evolving eigenfunction.

Proof. This follows immediately from the previous Proposition.

Now one can simply observe that the right-hand-side of (2.9) continues to make sense when passing from commutative to noncommutative tori, hence it can be taken as the appropriate extension of the right-hand-side of (1.3) in this context.

Thus, we conclude that a good analog of Ricci flow on noncommutative tori is provided by the equation (1.1).

In the following, we obtain a more explicit expression for the right-hand-side of (2.9) in the case of noncommutative tori, in terms of pseudodifferential calculus and the modular operator, as in [20]. 


\section{VALUE AT THE ORIGIN}

We first express the value $\zeta_{a}(0)$ in terms of pseudodifferential calculus, as in [20].

Proposition 3.1. Consider a pseudodifferential operator with symbol

$$
\sum_{j \geq 0} b_{j}\left(\xi_{1}, \xi_{2}\right)
$$

which is an approximation to the inverse of the operator $\left(\triangle^{\prime}+1\right)$ in the space of symbols, with $\left(\xi_{1}, \xi_{2}\right)$ the canonical coordinate system of $\mathbb{R}^{2}$. Here $b_{j}\left(\xi_{1}, \xi_{2}\right)$ is homogeneous of order $-2-j$. Then

$$
\zeta_{a}(0)=-\int \tau\left(a b_{2}\left(\xi_{1}, \xi_{2}\right)\right) d \xi_{1} d \xi_{2}=-\tau\left(a \int b_{2}\left(\xi_{1}, \xi_{2}\right) d \xi_{1} d \xi_{2}\right) .
$$

Proof. The pseudodifferential calculus for noncommutative tori associates to a symbol $\sigma(\xi)=\sum_{|j| \leq n} a_{j} \xi_{1}^{j_{1}} \xi_{2}^{j_{2}}$, with $a_{j} \in \mathcal{A}_{\theta}^{\infty}$ a differential operator $P_{\sigma}=\sum_{|j| \leq n} a_{j} \delta_{1}^{j_{1}} \delta_{2}^{j_{2}}$ and, more generally, a pseudodifferential operator to an arbitrary symbol $\sigma \in \mathcal{S}=$ $\cup_{n \in \mathbb{Z}} \mathcal{S}_{n}$, where $\mathcal{S}_{n}$ denotes the symbols of order $n$ as in Definition 4.1 of [20].

For the Laplacian $\triangle^{\prime} \sim k \triangle k$, under the assumption that $\operatorname{Ker}\left(\triangle^{\prime}\right)=0$, we can use Mellin transform to get

$$
\zeta(s)=\operatorname{Tr}\left(\triangle^{\prime-s}\right)=\frac{1}{\Gamma(s)} \int_{0}^{\infty} \operatorname{Tr}\left(e^{-t \triangle^{\prime}}\right) t^{s-1} d t
$$

Using symbols, one can express the zeta function as an integration

$$
\zeta(s)=\frac{1}{\Gamma(s)} \int_{0}^{\infty} \int \tau\left(\sigma\left(e^{-t \triangle^{\prime}}\right)(\xi)\right) t^{s-1} d \xi d t .
$$

If $\operatorname{Ker}(\triangle) \neq 0$, one replaces $\operatorname{Tr}\left(e^{-t \Delta^{\prime}}\right)$ with $\operatorname{Tr}^{+}\left(e^{-t \Delta^{\prime}}\right)=\operatorname{Tr}\left(e^{-t \triangle^{\prime}}\right)-\operatorname{dim} \operatorname{Ker}\left(\triangle^{\prime}\right)$.

We can proceed in a similar way for the zeta functions $\zeta_{a}(s)=\operatorname{Tr}\left(a \triangle^{\prime-s}\right)$. The meromorphic extension of the function $\zeta_{a}(s)$ to $\mathbb{C}$ is equivalent ([53] $\left.\S 3.3 .2 .1-3.3 .2 .2\right)$ to the existence of an asymptotic expansion for the resolvent traces $\operatorname{Tr}\left(a \partial_{\lambda}^{p}\left(\triangle^{\prime}-\right.\right.$ $\lambda)^{-1}$ ). Thus, by

$$
e^{-t \triangle^{\prime}}=\int_{C} e^{-\lambda}\left(t \triangle^{\prime}-\lambda\right)^{-1} d \lambda=t^{-p-1} \int_{C} e^{-\lambda} \partial_{\lambda t^{-1}}^{p}\left(\triangle^{\prime}-\lambda t^{-1}\right)^{-1} d \lambda,
$$

the generalized heat trace $\operatorname{Tr}\left(a e^{-t \triangle^{\prime}}\right)$ also has an asymptotic expansion in the limit $t \rightarrow 0^{+}$, which we can write as

$$
\operatorname{Tr}\left(a e^{-t \triangle^{\prime}}\right) \sim t^{-1} \sum_{n=0}^{\infty} B_{2 n}\left(a, \triangle^{\prime}\right) t^{n} .
$$

The zeta function $\zeta_{a}(s)$ and the generalized heat trace are related by the general heat-zeta transition formulae $([53, \S 3.3 .3 .2)$ using Mellin transform

$$
\zeta_{a}(s)=\operatorname{Tr}\left(a \triangle^{\prime-s}\right)=\frac{1}{\Gamma(s)} \int_{0}^{\infty} \operatorname{Tr}\left(a e^{-t \triangle^{\prime}}\right) t^{s-1} d t
$$

and

$$
\operatorname{Tr}\left(a e^{-t \triangle^{\prime}}\right)=\frac{1}{2 \pi i} \int_{\operatorname{Re}(s)=c} t^{-s} \zeta_{a}(s) \Gamma(s) d s .
$$

Here one replaces $\operatorname{Tr}\left(a e^{-t \triangle^{\prime}}\right)$ with $\operatorname{Tr}^{+}\left(a e^{-t \triangle^{\prime}}\right)$ if triviality of the kernel of $\triangle^{\prime}$ is not assumed. 
The very general Grubb-Seeley transition formulae (53] §3.3.3.2) relate an asymptotic expansion

$$
\epsilon(t) \sim \sum_{j=0}^{\infty} \sum_{\ell=0}^{n_{j}} a_{j, \ell} t^{\beta_{j}} \log ^{\ell}(t)
$$

for an increasing sequence of $0<\beta_{j} \rightarrow \infty$ and with $n_{j} \in \mathbb{N}$, to the meromorphic structure of the Mellin transform $f(z)=\int_{0}^{\infty} t^{z-1} \epsilon(t) d t$, by

$$
f(z) \sim \sum_{j=0}^{\infty} \sum_{\ell=0}^{n_{j}} \frac{(-1)^{\ell} \ell ! a_{j, \ell}}{\left(z+\beta_{j}\right)^{\ell+1}}
$$

When applied to the particular case (and simpler form) of the asymptotic expansion (3.3), considering the fact that the Gamma function has a simple pole at zero, we get

$$
\zeta_{a}(0)=B_{2}\left(a, \triangle^{\prime}\right) .
$$

Equivalently, using the symbols calculus, we have

$$
\frac{1}{\Gamma(s)} \int_{0}^{\infty} \operatorname{Tr}\left(a e^{-t \Delta^{\prime}}\right) t^{s-1} d t=\frac{1}{\Gamma(s)} \int_{0}^{\infty} \int \tau\left(a \sigma\left(e^{-t \triangle^{\prime}}\right)(\xi)\right) t^{s-1} d \xi d t,
$$

so that

$$
\zeta_{a}(0)=\operatorname{Res}_{s=0} \int_{0}^{\infty} \int \tau\left(a \sigma\left(e^{-t \triangle^{\prime}}\right)(\xi)\right) t^{s-1} d \xi d t,
$$

where, for $t \rightarrow 0^{+}$,

$$
\int \tau\left(a \sigma\left(e^{-t \triangle^{\prime}}\right)(\xi)\right) d \xi \sim t^{-1} \sum_{n=0}^{\infty} B_{2 n}\left(a, \triangle^{\prime}\right) t^{n} .
$$

Using again the Cauchy integral formula

$$
e^{-t \triangle^{\prime}}=\frac{1}{2 \pi i} \int_{C} e^{-t \lambda}\left(\triangle^{\prime}-\lambda\right)^{-1} d \lambda
$$

and approximating the inverse $\left(\triangle^{\prime}-\lambda\right)^{-1}$ by a pseudodifferential operator $B_{\lambda}$ with symbol (3.1), as in [20] and [29], one then finds

$$
B_{2}\left(a, \triangle^{\prime}\right)=\frac{1}{2 \pi i} \int_{C} e^{-\lambda} \tau\left(a b_{2}(\xi, \lambda)\right) d \lambda d \xi
$$

and, by the same homogeneity argument used in [20] and [29], one then finds, as in (3.2) that $\zeta_{a}(0)=-\tau\left(a \int b_{2}(\xi) d \xi\right)$.

We can then rewrite the above result, using the following computation as in [20].

Lemma 3.2. Consider the pseudodifferential operator with symbol (3.1) as above. Then

$$
\int b_{2}\left(\xi_{1}, \xi_{2}\right) d \xi_{1} d \xi_{2}=2 \pi \int_{0}^{\infty} T(r) b_{0} r d r
$$


where $b_{0}=\left(k^{2} r^{2}+1\right)^{-1}$ and $T(r)$ is given by the explicit expression (using Einstein's summation notation)

$$
\begin{aligned}
T(r) & =-b_{0} k \delta_{i} \delta_{i}(k)+3 r^{2} k^{3} b_{0}^{2} \delta_{i} \delta_{i}(k)+4 r^{2} k^{2} b_{0}^{2} \delta_{i}(k) \delta_{i}(k)+r^{2} k^{2} b_{0}^{2} \delta_{i} \delta_{i}(k) k \\
& -2 r^{4} k^{5} b_{0}^{3} \delta_{i} \delta_{i}(k)-4 r^{4} k^{4} b_{0}^{3} \delta_{i}(k) \delta_{i}(k)-2 r^{4} k^{4} b_{0}^{3} \delta_{i} \delta_{i}(k) k \\
& +r^{2} k b_{0} \delta_{i}(k) b_{0}\left(4 k \delta_{i}(k)+2 \delta_{i}(k) k\right)-2 r^{4} k b_{0} \delta_{i}(k) k^{2} b_{0}^{2}\left(k \delta_{i}(k)+\delta_{i}(k) k\right) \\
& -r^{4} k^{3} b_{0}^{2} \delta_{i}(k) b_{0}\left(8 k \delta_{i}(k)+6 \delta_{i}(k) k\right)+2 r^{6} k^{3} b_{0}^{2} \delta_{i}(k) k^{2} b_{0}^{2}\left(k \delta_{i}(k)+\delta_{i}(k) k\right) \\
& -r^{4} k^{2} b_{0}^{2} \delta_{i}(k) k b_{0}\left(6 k \delta_{i}(k)+4 \delta_{i}(k) k\right)+2 r^{6} k^{2} b_{0}^{2} \delta_{i}(k) k^{3} b_{0}^{2}\left(k \delta_{i}(k)+\delta_{i}(k) k\right) \\
& +4 r^{6} k^{5} b_{0}^{3} \delta_{i}(k) b_{0}\left(k \delta_{i}(k)+\delta_{i}(k) k\right)+4 r^{6} k^{4} b_{0}^{3} \delta_{i}(k) k b_{0}\left(k \delta_{i}(k)+\delta_{i}(k) k\right) .
\end{aligned}
$$

Proof. In [20] it is shown that

$$
\int b_{2}\left(\xi_{1}, \xi_{2}\right) d \xi_{1} d \xi_{2}=\int S\left(\xi_{1}, \xi_{2}\right) b_{0} d \xi_{1} d \xi_{2}
$$

where $b_{0}=\left(k^{2} \xi_{1}^{2}+k^{2} \xi_{2}^{2}+1\right)^{-1}$ and $S\left(\xi_{1}, \xi_{2}\right)$ is given by the explicit expression (6.1) reported in the Appendix below. After passing to polar coordinates in (6.1) we obtain (3.7).

3.1. The modular operator. For consistency, we will be following here the somewhat unfortunate choice of notation adopted in [20], according to which we will continue to denote the Laplacian by $\triangle$, while at the same time using the very similar notation $\Delta$ for the modular operator.

The latter is defined by

$$
\Delta(x)=e^{-h} x e^{h}, \quad \text { for } \quad x \in \mathcal{A}_{\theta}^{\infty},
$$

for $h$ as in (2.3).

We recall the following result on the modular operator $\Delta$.

Lemma 3.3. (Connes-Tretkoff [20]) For every element $\rho$ of $\mathcal{A}_{\theta}^{\infty}$ and every nonnegative integer $m$ one has

$$
\int_{0}^{\infty} \frac{k^{2 m+2} u^{m}}{\left(k^{2} u+1\right)^{m+1}} \rho \frac{1}{\left(k^{2} u+1\right)} d u=\mathcal{D}_{m}(\rho)
$$

where

$$
\mathcal{D}_{m}=\int_{0}^{\infty} \frac{x^{m}}{(x+1)^{m+1}} \frac{1}{(x \Delta+1)} d x .
$$

We refer the reader to [20] for more details. In the same way, we obtain the following variant.

Proposition 3.4. For $\lambda, \rho \in \mathcal{A}_{\theta}^{\infty}$ and $w, y, v \in \mathbb{Z}_{>0}$,

$$
\int_{0}^{\infty} \frac{1}{\left(k^{2} u+1\right)^{v}} \lambda \frac{k^{2 w} u^{(w-1)}}{\left(k^{2} u+1\right)^{y}} \rho \frac{1}{\left(k^{2} u+1\right)} d u=D_{w, v, y}(\lambda, \rho)
$$

where

$$
D_{w, v, y}(\lambda, \rho)=\int_{0}^{\infty}\left[\frac{1}{\left(x \Delta^{-1}+1\right)^{v}}(\lambda)\right] \frac{x^{w-1}}{(x+1)^{y}}\left[\frac{1}{(x \Delta+1)}(\rho)\right] d x
$$


Proof. Using $k=e^{h / 2}$ and the change of variable $u=e^{s}$, we get

$$
\begin{gathered}
\int_{0}^{\infty} \frac{1}{\left(k^{2} u+1\right)^{v}} \lambda \frac{k^{2 w} u^{(w-1)}}{\left(k^{2} u+1\right)^{y}} \rho \frac{1}{\left(k^{2} u+1\right)} d u \\
=\int_{-\infty}^{\infty} \frac{1}{\left(e^{(s+h)}+1\right)^{v}} \lambda \frac{e^{h w+s(w-1)}}{\left(e^{(s+h)}+1\right)^{y}} \rho \frac{e^{s}}{\left(e^{(s+h)}+1\right)} d s \\
=\int_{-\infty}^{\infty} \frac{e^{(s+h) / 2}}{\left(e^{(s+h)}+1\right)^{v}} \Delta^{1 / 2}(\lambda) \frac{e^{(s+h)(w-1)}}{\left(e^{(s+h)}+1\right)^{y}} \Delta^{-1 / 2}(\rho) \frac{e^{(s+h) / 2}}{\left(e^{(s+h)}+1\right)} d s \\
=\int_{-\infty}^{\infty} \frac{1}{\left(e^{(s+h)}+1\right)^{(v-1)}} \Upsilon(h, s) \Delta^{1 / 2}(\lambda) \frac{e^{(s+h)(w-1)}}{\left(e^{(s+h)}+1\right)^{y}} \Delta^{-1 / 2}(\rho) \Upsilon(h, s) d s,
\end{gathered}
$$

where

$$
\Upsilon(h, s)=\int_{-\infty}^{\infty} \frac{e^{i t(s+h)}}{\left(e^{\pi t}+e^{-\pi t}\right)} d t
$$

We then further write the above as

$$
=\int_{-\infty}^{\infty} \frac{1}{\left(e^{(s+h)}+1\right)^{(v-1)}} \Xi_{1,(h, s)}(\lambda) \frac{e^{(s+h)(w-1)}}{\left(e^{(s+h)}+1\right)^{y}} \Lambda_{(h, s)}(\rho) d s,
$$

where

$$
\begin{aligned}
& \Xi_{1,(h, s)}(\lambda)=\int_{-\infty}^{\infty} \Delta^{1 / 2-i t}(\lambda) \frac{e^{i t(s+h)}}{\left(e^{\pi t}+e^{-\pi t}\right)} d t \\
& \Lambda_{(h, s)}(\rho)=\int_{-\infty}^{\infty} \frac{e^{i t(s+h)}}{\left(e^{\pi t}+e^{-\pi t}\right)} \Delta^{-1 / 2+i t}(\rho) d t .
\end{aligned}
$$

We can then proceed by induction to obtain

$$
=\int_{-\infty}^{\infty} \Xi_{v,(h, s)}(\lambda) \frac{e^{(s+h)(w-1)}}{\left(e^{(s+h)}+1\right)^{y}} \Lambda_{(h, s)}(\rho) d s,
$$

with $\Lambda_{(h, s)}(\rho)$ as above and with

$$
\Xi_{v,(h, s)}(\lambda)=\prod_{j=1}^{v} \int_{-\infty}^{\infty} \Delta^{1 / 2-i t_{j}}(\lambda) \frac{e^{i t_{j}(s+h)}}{\left(e^{\pi t_{j}}+e^{-\pi t_{j}}\right)} d t_{j} .
$$

We further write the above as

$$
\begin{aligned}
& =\int_{-\infty}^{\infty} \int_{-\infty}^{\infty} \cdots \int_{-\infty}^{\infty}\left(\prod_{j=1}^{v} \Delta^{1 / 2-i t_{j}}(\lambda) \frac{e^{i t_{j} h}}{\left(e^{\pi t_{j}}+e^{-\pi t_{j}}\right)}\right) \\
& \quad \times\left(\int_{-\infty}^{\infty} \frac{e^{(s+h)(w-(v+1) / 2)}\left(\left(e^{i t s} \prod_{j=1}^{v} e^{i t_{j} s}\right)\right)}{\left(e^{(s+h)}+1\right)^{y}} d s\right) \\
& \quad \times \frac{e^{i t h}}{\left(e^{\pi t}+e^{-\pi t}\right)} \Delta^{-1 / 2+i t}(\rho) d t d t_{1} \cdots d t_{v} .
\end{aligned}
$$

We denote by $F_{w, v, y}$ the Fourier transform of the function

$$
h_{w, v, y}(s)=\frac{e^{s(w-(v+1) / 2)}}{\left(e^{s}+1\right)^{y}} .
$$


The expression above then becomes

$$
\begin{aligned}
& =\int_{-\infty}^{\infty} \int_{-\infty}^{\infty} \cdots \int_{-\infty}^{\infty}\left(\prod_{j=1}^{v} \Delta^{1 / 2-i t_{j}}(\lambda) \frac{1}{\left(e^{\pi t_{j}}+e^{-\pi t_{j}}\right)}\right) \\
& \quad \times F_{w, v, y}\left(t+\sum_{j=1}^{v} t_{j}\right) \frac{1}{\left(e^{\pi t}+e^{-\pi t}\right)} \Delta^{-1 / 2+i t}(\rho) d t d t_{1} \cdots d t_{v} \\
& =\int_{0}^{\infty}\left[\left(\frac{x^{1 / 2}}{x \Delta^{-1}+1}\right)^{v}(\lambda)\right] \frac{x^{(w-(v+1) / 2)}}{(x+1)^{y}}\left[\left(\frac{x^{1 / 2}}{x \Delta+1}\right)(\rho)\right] \frac{d x}{x} \\
& =\int_{0}^{\infty}\left[\frac{1}{\left(x \Delta^{-1}+1\right)^{v}}(\lambda)\right] \frac{x^{w-1}}{(x+1)^{y}}\left[\frac{1}{(x \Delta+1)}(\rho)\right] d x .
\end{aligned}
$$

This is the expression $D_{w, v, y}(\lambda, \rho)$ of (3.13).

We can now conclude the argument and give an expression for the Ricci flow in terms of these properties of the modular operator.

Theorem 3.5. The Ricci flow on the noncommutative torus is given by

$$
\frac{d \lambda}{d t}=\lambda 12 \pi \zeta_{g^{2}}(0)=\lambda \tau\left(f^{2} \mathcal{R}\right),
$$

where $\lambda$ and $f$ represent (respectively) the evolving eigenvalues and the corresponding evolving normalized eigenfunctions of the Laplacian $\triangle^{\prime} \sim k \triangle k$ and

$$
\begin{aligned}
\frac{-1}{12 \pi^{2}} \mathcal{R} & =\sum_{i=1,2}\left(-k^{-1} \mathcal{D}_{0}\left(\delta_{i}^{2}(k)\right)+3 k^{-1} \mathcal{D}_{1}\left(\delta_{i}^{2}(k)\right)\right. \\
& +4 k^{-2} \mathcal{D}_{1}\left(\delta_{i}(k)^{2}\right)+k^{-2} \mathcal{D}_{1}\left(\delta_{i}^{2}(k) k\right)-2 k^{-1} \mathcal{D}_{2}\left(\delta_{i}^{2}(k)\right) \\
& -4 k^{-2} \mathcal{D}_{2}\left(\delta_{i}(k)^{2}\right)-2 k^{-2} \mathcal{D}_{2}\left(\delta_{i}^{2}(k) k\right) \\
& +k D_{2,1,1}\left(\delta_{i}(k) k^{-4}, 4 k \delta_{i}(k)+2 \delta_{i}(k) k\right) \\
& -2 k D_{3,1,2}\left(\delta_{i}(k) k^{-4}, k \delta_{i}(k)+\delta_{i}(k) k\right) \\
& -k^{3} D_{3,2,1}\left(\delta_{i}(k) k^{-6}, 8 k \delta_{i}(k)+6 \delta_{i}(k) k\right) \\
& +2 k^{3} D_{4,2,2}\left(\delta_{i}(k) k^{-6}, k \delta_{i}(k)+\delta_{i}(k) k\right) \\
& -k^{2} D_{3,2,1}\left(\delta_{i}(k) k^{-5}, 6 k \delta_{i}(k)+4 \delta_{i}(k) k\right) \\
& +2 k^{2} D_{4,2,2}\left(\delta_{i}(k) k^{-5}, k \delta_{i}(k)+\delta_{i}(k) k\right) \\
& +4 k^{5} D_{4,3,1}\left(\delta_{i}(k) k^{-8}, k \delta_{i}(k)+\delta_{i}(k) k\right) \\
& \left.+4 k^{4} D_{4,3,1}\left(\delta_{i}(k) k^{-7}, k \delta_{i}(k)+\delta_{i}(k) k\right)\right),
\end{aligned}
$$

with $k=e^{h / 2}, h=h^{*} \in \mathcal{A}_{\theta}^{\infty}$, and $\Delta$ defined by $\Delta(x)=e^{-h} x e^{h}$ for $x \in \mathcal{A}_{\theta}^{\infty}$, and with

$$
\begin{gathered}
\mathcal{D}_{m}=\int_{0}^{\infty} \frac{x^{m}}{(x+1)^{m+1}} \frac{1}{(x \Delta+1)} d x \\
D_{w, v, y}(\gamma, \rho)=\int_{0}^{\infty}\left[\frac{1}{\left(x \Delta^{-1}+1\right)^{v}}(\gamma)\right] \frac{x^{w-1}}{(x+1)^{y}}\left[\frac{1}{(x \Delta+1)}(\rho)\right] d x,
\end{gathered}
$$

for $\gamma, \rho \in \mathcal{A}_{\theta}^{\infty}$. 
Proof. In Lemma 3.2 we have

with

$$
\zeta_{a}(0)=\frac{1}{12 \pi} \tau(a \mathcal{R})
$$

$$
\frac{-1}{12 \pi^{2}} \mathcal{R}=2 \int_{0}^{\infty} T(r) b_{0} r d r=\int_{0}^{\infty} T(r) b_{0} 2 r d r
$$

Using Lemma 3.3 and Proposition 3.4, with the change of variable $u=r^{2}$, we can rewrite the explicit expression (3.7) for $T(r)$ and obtain the result as stated.

\section{Noncommutative Black HOLES}

We give here an illustration of a possible physical application of the Ricci flow on noncommutative tori discussed in the previous sections.

4.1. The BTZ black hole. The Bañados-Teitelboim-Zanelli (BTZ) black hole [3] is a black hole in $(2+1)$-dimensional gravity. It is an asymptotically $\mathrm{AdS}_{2+1}$ space obtained as a global quotient of anti-de Sitter space by a discrete group of isometries $\Gamma \subset S O(2,2)$ generated by a single loxodromic element. In Euclidean gravity, the Euclidean version of the BTZ black hole is given by a quotient $\mathcal{X}_{q}=\mathbb{H}^{3} /\left(q^{\mathbb{Z}}\right)$ of 3-dimensional real hyperbolic space $\mathbb{H}^{3}$ (the Euclidean version of $\mathrm{AdS}_{2+1}$ ) by a subgroup $\Gamma=q^{\mathbb{Z}}$ of $\operatorname{PSL}(2, \mathbb{C})$ generated by an element $q \in \mathbb{C}^{*}$ with $|q|<1$, acting on $\mathbb{H}^{3}$ by $(z, y) \mapsto(q z,|q| y)$ in the realization of $\mathbb{H}^{3}$ as the upper half space $\mathbb{C} \times \mathbb{R}_{+}^{*}$, see [7, 39], [45. The space $\mathcal{X}_{q}$ obtained in this way describes a spinning black hole whenever $q$ is not purely real. Topologically $\mathcal{X}_{q}$ is a solid torus, endowed with a hyperbolic metric with conformal boundary at infinity given by the Tateuniformized elliptic curve $E_{q}(\mathbb{C})=\mathbb{C}^{*} /\left(q^{\mathbb{Z}}\right)$. In other words, the compactification $\overline{\mathcal{X}}_{q}=\mathcal{X}_{q} \cup E_{q}(\mathbb{C})$ is the quotient $\Omega_{\Gamma} / \Gamma$ of the action of $\Gamma=q^{\mathbb{Z}}$ on the domain of discontinuity $\Omega_{\Gamma} \subset \mathbb{H}^{3} \cup \mathbb{P}^{1}(\mathbb{C})$, that is, the subset of the compactified $\overline{\mathbb{H}^{3}}=\mathbb{H}^{3} \cup$ $\mathbb{P}^{1}(\mathbb{C})$ on which $\Gamma$ acts freely and properly discontinuously. This is the complement of the limit set $\Lambda_{\Gamma}=\{0, \infty\} \subset \mathbb{P}^{1}(\mathbb{C})$.

In recent years, noncommutative deformations of the BTZ black hole were considered in the context of string theory compactifications and of various proposals for noncommutative models of gravity, see for instance [5], 26], 27].

4.2. From elliptic curves to noncommutative tori. We consider here a very natural noncommutative deformation of the Euclidean BTZ black hole, based on considering a degenerating family of BTZ black holes, where the uniformization parameter $q$ tends to a point on the unit circle with irrational angle $q \rightarrow e^{2 \pi i \theta}$, with $\theta \in \mathbb{R} \backslash \mathbb{Q}$. This has the effect of replacing the elliptic curve $E_{q}(\mathbb{C})=\mathbb{C}^{*} /\left(q^{\mathbb{Z}}\right)$ with a "bad quotient" described by (the suspension of) a noncommutative torus with algebra of functions $C\left(S^{1}\right) \rtimes_{\theta} \mathbb{Z}=\mathcal{A}_{\theta}$. The suspension corresponds to the fact that the group $\Gamma=e^{2 \pi i \theta \mathbb{Z}}$ now acts trivially on the radial direction in $\mathbb{C}^{*}=S^{1} \times R_{+}^{*}$ so that the crossed product algebra describing the quotient is given by $\mathcal{A}_{\theta} \otimes C_{0}\left(\mathbb{R}_{+}^{*}\right)$.

Notice how in this case, while one thinks of the noncommutative torus itself as a 2-dimensional geometry, in the degeneration of the elliptic curve $E_{q}(\mathbb{C})$ when $q \rightarrow$ $e^{2 \pi i \theta}$, it accounts only for a 1-dimensional geometry, which is the "bad quotient" of a circle by the action of $\Gamma=e^{2 \pi i \theta \mathbb{Z}} \simeq \mathbb{Z}$ by irrational rotations. One is left with a commutative radial direction $\mathbb{R}_{+}^{*}$ which is not affected by the quotient, since 
it is acted upon trivially. From the point of view of Ricci flow, this now allows for more interesting behaviors than in the original commutative case, intuitively because a one-dimensional geometry has been replaced in the limit by a richer two-dimensional (but noncommutative) geometry. We discuss this briefly in 84.3 below.

As the horizon $E_{q}(\mathbb{C})$ degenerates in this way to a noncommutative torus $\mathbb{T}_{\theta}^{2}$, described in terms of the algebra $\mathcal{A}_{\theta} \otimes C_{0}\left(\mathbb{R}_{+}^{*}\right)$ above, the bulk space $\mathcal{X}_{q}$ also is correspondingly deformed to a noncommutative space. In the limit $q \rightarrow e^{2 \pi i \theta}$ the action on $\mathbb{H}^{3}$ becomes $(z, y) \mapsto\left(e^{2 \pi i \theta} z, y\right)$. The resulting "bad quotient" $\mathcal{X}_{\theta}$ exists as a nice classical space and is replaced by a noncommutative space, which one can think of intuitively as a foliation by noncommutative tori. In fact, we have now the domain of discontinuity replaced by the set $\Omega_{\theta}=\overline{H^{3}} \backslash\{z=0\}$ on which $\Gamma=e^{2 \pi i \theta \mathbb{Z}}$ acts by $(z, y) \mapsto\left(e^{2 \pi i \theta} z, y\right)$. All the bad quotients $\left\{(z, y): z \in \mathbb{C}^{*}, y=y_{0}\right\} / \Gamma$ are (suspensions of) noncommutative tori $\mathbb{T}_{\theta}^{2}$ and the action of $\Gamma$ is trivial along the vertical direction, so we can regard the resulting space $\mathcal{X}_{\theta}$ as a one-parameter family of noncommutative spaces $\mathbb{T}_{\theta}^{2} \times \mathbb{R}_{+}^{*}$, one over each point $y$ in the vertical direction. More precisely, one obtains in this description an algebra of coordinates that is of the form $\mathcal{A}_{\theta} \otimes C_{0}\left(\mathbb{R}_{+}^{*}\right) \otimes C_{0}\left(\mathbb{R}_{+}^{*}\right)$, where the further commutative direction $\mathbb{R}_{+}^{*}$ corresponds to the vertical direction in $\mathbb{H}^{3}$. We can identify $C_{0}\left(\mathbb{R}_{+}^{*}\right) \otimes C_{0}\left(\mathbb{R}_{+}^{*}\right)$ with $C_{0}\left(\mathbb{R}_{+}^{*} \times \mathbb{R}_{+}^{*}\right)$ and further $C_{0}\left(\mathbb{R}^{2}\right)$ by a homeomorphic change of coordinates $\mathbb{R}_{+}^{*} \ni r=e^{\rho}, \rho \in \mathbb{R}$. This non-unital algebra $C_{0}\left(\mathbb{R}^{2}\right)$ has a unitization given by $C\left(S^{2}\right)$, by passing to the one point compactification.

4.3. Noncommutative black holes. We have described above a noncommutative deformation of the Euclidean BTZ black hole at a purely topological level (as a $C^{*}$ algebra). We now consider the metric aspect and the behavior under the Ricci flow. First notice that the original BTZ black hole, as a finite quotient of $\mathbb{H}^{3}$ by a discrete group of isometries, inherits from $\mathbb{H}^{3}$ the hyperbolic metric. On an $n$-dimensional hyperbolic space $\mathbb{H}^{n}$, with the standard hyperbolic metric, the Ricci tensor satisfies $\operatorname{Ric}(g)=-(n-1) g$, hence it flows as $g(t)=(1+2(n-1) t) g$, expanding for all times, with a backward blowup at $t=-1 /(2(n-1))$.

In the noncommutative deformation described above, one obtains different behaviors under the Ricci flow, depending on how one treats the topology of the commutative directions. As a product geometry $\mathbb{T}_{\theta}^{2} \times \mathbb{R}^{2}$, we can regard it as endowed with a product metric. In that case, the Ricci flow preserves this form and each factor evolves with its own Ricci flow equation. Thus, the noncommutative tori evolve according to the Ricci flow equation we described in the previous section, while for the commutative direction we can have, for example, shrinking spherical geometries, when compactifying to the one-point compactification $S^{2}$, steady torus geometries if compactifying to an $S^{1} \times S^{1}$, or more interesting possibilities such as a Witten black hole, also known as a Hamilton cigar soliton, see [14 §4.3. This latter possibility is physically more suitable for a black hole interpretation of the resulting space. A more interesting class of behaviors would be obtained if, instead of the product metric, one would consider a warped product, where the metric on the noncommutative torus $\mathbb{T}_{\theta}^{2}$ (that is, the element $k=e^{h / 2}$ with $h=h^{*} \in \mathcal{A}_{\theta}^{\infty}$ ) varies as a smooth function of the point in $\mathbb{R}^{2}$. 


\section{Directions AND PERspectives}

The formulation of the Ricci flow we proposed here for 2-dimensional noncommutative tori is based on the behavior of eigenvalues of the Laplacian on a 2dimensional manifold, under the Ricci flow, assuming some regularity conditions on the evolving eigenvalues and eigenfunctions as in [25]. In the case of classical 2-dimensional tori one knows 33 that an arbitrary initial metric flows to the flat metric under the Ricci flow. The expression we obtained in Theorem 3.5 appears to be, in its present form, too complicated to check directly whether this same property continues to hold for the noncommutative tori, but we expect that further investigation along these lines will answer this natural question.

In general, we hope that a good understanding of the Ricci flow on the noncommutative two torus would lead us to a deeper understanding of the geometry of spectral triples and the underlying topology, as did the original Ricci flow for the classical case.

Whether a similar spectral formulation of the Ricci flow can be given for a larger class of noncommutative spaces appears to be another interesting question. The Ricci flow has been successfully applied in understanding the relations between geometric and topological properties of Riemannian manifolds, in particular 3 -manifolds. A noncommutative analog of the Ricci flow would allow us to pursue the same idea regarding this larger class of geometries.

In dimension higher than two, the equation obtained in 25] for the Ricci flow (under the same strong assumptions) has the more complicated form

$$
\frac{d \lambda}{d t}=\lambda \int_{M} f^{2} R d \mu-\int_{M} R|\nabla f|^{2} d \mu+2 \int_{M} \operatorname{Ric}(\nabla f, \nabla f) d \mu,
$$

for the evolving Laplace eigenvalues and the corresponding normalized evolving eigenfunctions. Thus, if one wishes to extend the approach proposed here, one needs to provide a suitable interpretation of these additional terms, presumably in terms of the heat-kernel expansion of a suitable Laplacian operator on the noncommutative geometry. A good model case on which to test this method in dimension three, where the Ricci flow should be most interesting, may be the rich collection of noncommutative 3 -spheres, whose geometry and moduli spaces were studied by Connes and Dubois-Violette in [19.

Another possible point of view, which is naturally suggested by the case we considered here, is the relation between the Ricci flow and the Yamabe flow in 2-dimensions. This means that gaining a good understanding of how to extend the Ricci flow to 2-dimensional noncommutative spaces, such as the noncommutative tori, may also yield useful suggestions on how to extend to noncommutative geometry the Yamabe flow and the Yamabe problem in higher dimensions. Also, by working with the determinant of the Laplacian and its evolution equation under the Ricci flow, one would, by similar techniques, be able to extend to noncommutative 2-tori the results of [40] and obtain an analog of the Osgood-Phillips-Sarnak theorem in this setting, 48].

In terms of other possible physical applications, it is well known (28, see also 11] for a detailed discussion) that the Ricci flow arises as the weak coupling limit of the renormalization group flow for non-linear sigma models. In recent years, generalizations of non-linear sigma models were considered, where the both the target and the source space can be replaced by noncommutative spaces. In particular, [46] 
gives a general form for an action functional where the target space is a noncommutative Riemannian manifold (a spectral triple) and the source space can also be a noncommutative space. In particular, they study the case of sigma-models where these spaces are noncommutative tori. It would be interesting to relate Ricci flow on noncommutative tori considered here to the renormalization group analysis of these sigma-models.

Acknowledgment. This paper is based on the results of the first author's summer research project, within the Caltech program Summer Undergraduate Research Fellowships (SURF). The first author acknowledges support given from the bequest of Herbert J. Ryser (1923-1985) through the Caltech mathematics department. The second author acknowledges support from NSF grants DMS-0901221, DMS-1007207.

\section{Appendix}

We report here, for the convenience of the reader, the explicit expression obtained in 20] for $S\left(\xi_{1}, \xi_{2}\right)$ in (3.8), which we used in 93 above:

$$
\begin{aligned}
& S\left(\xi_{1}, \xi_{2}\right)= \\
& -b_{0} k \delta_{1}^{2}(k)-b_{0} k \delta_{2}^{2}(k)+\left(\xi_{2}^{2}+5 \xi_{1}^{2}\right)\left(k^{2} b_{0}^{2}\right) k \delta_{1}^{2}(k)+\left(5 \xi_{2}^{2}+\xi_{1}^{2}\right)\left(k^{2} b_{0}^{2}\right) k \delta_{2}^{2}(k)+2 \xi_{2}^{2}\left(k^{2} b_{0}^{2}\right) \delta_{1}(k) \delta_{1}(k)+ \\
& 6 \xi_{1}^{2}\left(k^{2} b_{0}^{2}\right) \delta_{1}(k) \delta_{1}(k)+\xi_{2}^{2}\left(k^{2} b_{0}^{2}\right) \delta_{1}^{2}(k) k+\xi_{1}^{2}\left(k^{2} b_{0}^{2}\right) \delta_{1}^{2}(k) k+6 \xi_{2}^{2}\left(k^{2} b_{0}^{2}\right) \delta_{2}(k) \delta_{2}(k)+ \\
& 2 \xi_{1}^{2}\left(k^{2} b_{0}^{2}\right) \delta_{2}(k) \delta_{2}(k)+\xi_{2}^{2}\left(k^{2} b_{0}^{2}\right) \delta_{2}^{2}(k) k+\xi_{1}^{2}\left(k^{2} b_{0}^{2}\right) \delta_{2}^{2}(k) k-4 \xi_{2}^{2} \xi_{1}^{2}\left(k^{4} b_{0}^{3}\right) k \delta_{1}^{2}(k)- \\
& 4 \xi_{1}^{4}\left(k^{4} b_{0}^{3}\right) k \delta_{1}^{2}(k)-4 \xi_{2}^{4}\left(k^{4} b_{0}^{3}\right) k \delta_{2}^{2}(k)-4 \xi_{2}^{2} \xi_{1}^{2}\left(k^{4} b_{0}^{3}\right) k \delta_{2}^{2}(k)-8 \xi_{2}^{2} \xi_{1}^{2}\left(k^{4} b_{0}^{3}\right) \delta_{1}(k) \delta_{1}(k)- \\
& 8 \xi_{1}^{4}\left(k^{4} b_{0}^{3}\right) \delta_{1}(k) \delta_{1}(k)-4 \xi_{2}^{2} \xi_{1}^{2}\left(k^{4} b_{0}^{3}\right) \delta_{1}^{2}(k) k-4 \xi_{1}^{4}\left(k^{4} b_{0}^{3}\right) \delta_{1}^{2}(k) k-8 \xi_{2}^{4}\left(k^{4} b_{0}^{3}\right) \delta_{2}(k) \delta_{2}(k)- \\
& 8 \xi_{2}^{2} \xi_{1}^{2}\left(k^{4} b_{0}^{3}\right) \delta_{2}(k) \delta_{2}(k)-4 \xi_{2}^{4}\left(k^{4} b_{0}^{3}\right) \delta_{2}^{2}(k) k-4 \xi_{2}^{2} \xi_{1}^{2}\left(k^{4} b_{0}^{3}\right) \delta_{2}^{2}(k) k+2 \xi_{2}^{2} b_{0} k \delta_{1}(k) b_{0} k \delta_{1}(k)+ \\
& 6 \xi_{1}^{2} b_{0} k \delta_{1}(k) b_{0} k \delta_{1}(k)+2 \xi_{2}^{2} b_{0} k \delta_{1}(k) b_{0} \delta_{1}(k) k+2 \xi_{1}^{2} b_{0} k \delta_{1}(k) b_{0} \delta_{1}(k) k-4 \xi_{2}^{2} \xi_{1}^{2} b_{0} k \delta_{1}(k)\left(k^{2} b_{0}^{2}\right) k \delta_{1}(k)- \\
& 4 \xi_{1}^{4} b_{0} k \delta_{1}(k)\left(k^{2} b_{0}^{2}\right) k \delta_{1}(k)-4 \xi_{2}^{2} \xi_{1}^{2} b_{0} k \delta_{1}(k)\left(k^{2} b_{0}^{2}\right) \delta_{1}(k) k-4 \xi_{1}^{4} b_{0} k \delta_{1}(k)\left(k^{2} b_{0}^{2}\right) \delta_{1}(k) k+ \\
& 6 \xi_{2}^{2} b_{0} k \delta_{2}(k) b_{0} k \delta_{2}(k)+2 \xi_{1}^{2} b_{0} k \delta_{2}(k) b_{0} k \delta_{2}(k)+2 \xi_{2}^{2} b_{0} k \delta_{2}(k) b_{0} \delta_{2}(k) k+2 \xi_{1}^{2} b_{0} k \delta_{2}(k) b_{0} \delta_{2}(k) k- \\
& 4 \xi_{2}^{4} b_{0} k \delta_{2}(k)\left(k^{2} b_{0}^{2}\right) k \delta_{2}(k)-4 \xi_{2}^{2} \xi_{1}^{2} b_{0} k \delta_{2}(k)\left(k^{2} b_{0}^{2}\right) k \delta_{2}(k)-4 \xi_{2}^{4} b_{0} k \delta_{2}(k)\left(k^{2} b_{0}^{2}\right) \delta_{2}(k) k- \\
& 4 \xi_{2}^{2} \xi_{1}^{2} b_{0} k \delta_{2}(k)\left(k^{2} b_{0}^{2}\right) \delta_{2}(k) k-2 \xi_{2}^{4}\left(k^{2} b_{0}^{2}\right) k \delta_{1}(k) b_{0} k \delta_{1}(k)-16 \xi_{2}^{2} \xi_{1}^{2}\left(k^{2} b_{0}^{2}\right) k \delta_{1}(k) b_{0} k \delta_{1}(k)- \\
& 14 \xi_{1}^{4}\left(k^{2} b_{0}^{2}\right) k \delta_{1}(k) b_{0} k \delta_{1}(k)-2 \xi_{2}^{4}\left(k^{2} b_{0}^{2}\right) k \delta_{1}(k) b_{0} \delta_{1}(k) k-12 \xi_{2}^{2} \xi_{1}^{2}\left(k^{2} b_{0}^{2}\right) k \delta_{1}(k) b_{0} \delta_{1}(k) k- \\
& 10 \xi_{1}^{4}\left(k^{2} b_{0}^{2}\right) k \delta_{1}(k) b_{0} \delta_{1}(k) k+4 \xi_{2}^{4} \xi_{1}^{2}\left(k^{2} b_{0}^{2}\right) k \delta_{1}(k)\left(k^{2} b_{0}^{2}\right) k \delta_{1}(k)+8 \xi_{2}^{2} \xi_{1}^{4}\left(k^{2} b_{0}^{2}\right) k \delta_{1}(k)\left(k^{2} b_{0}^{2}\right) k \delta_{1}(k)+ \\
& 4 \xi_{1}^{6}\left(k^{2} b_{0}^{2}\right) k \delta_{1}(k)\left(k^{2} b_{0}^{2}\right) k \delta_{1}(k)+4 \xi_{2}^{4} \xi_{1}^{2}\left(k^{2} b_{0}^{2}\right) k \delta_{1}(k)\left(k^{2} b_{0}^{2}\right) \delta_{1}(k) k+8 \xi_{2}^{2} \xi_{1}^{4}\left(k^{2} b_{0}^{2}\right) k \delta_{1}(k)\left(k^{2} b_{0}^{2}\right) \delta_{1}(k) k+ \\
& 4 \xi_{1}^{6}\left(k^{2} b_{0}^{2}\right) k \delta_{1}(k)\left(k^{2} b_{0}^{2}\right) \delta_{1}(k) k-14 \xi_{2}^{4}\left(k^{2} b_{0}^{2}\right) k \delta_{2}(k) b_{0} k \delta_{2}(k)-16 \xi_{2}^{2} \xi_{1}^{2}\left(k^{2} b_{0}^{2}\right) k \delta_{2}(k) b_{0} k \delta_{2}(k)- \\
& 2 \xi_{1}^{4}\left(k^{2} b_{0}^{2}\right) k \delta_{2}(k) b_{0} k \delta_{2}(k)-10 \xi_{2}^{4}\left(k^{2} b_{0}^{2}\right) k \delta_{2}(k) b_{0} \delta_{2}(k) k-12 \xi_{2}^{2} \xi_{1}^{2}\left(k^{2} b_{0}^{2}\right) k \delta_{2}(k) b_{0} \delta_{2}(k) k- \\
& 2 \xi_{1}^{4}\left(k^{2} b_{0}^{2}\right) k \delta_{2}(k) b_{0} \delta_{2}(k) k+4 \xi_{2}^{6}\left(k^{2} b_{0}^{2}\right) k \delta_{2}(k)\left(k^{2} b_{0}^{2}\right) k \delta_{2}(k)+8 \xi_{2}^{4} \xi_{1}^{2}\left(k^{2} b_{0}^{2}\right) k \delta_{2}(k)\left(k^{2} b_{0}^{2}\right) k \delta_{2}(k)+ \\
& 4 \xi_{2}^{2} \xi_{1}^{4}\left(k^{2} b_{0}^{2}\right) k \delta_{2}(k)\left(k^{2} b_{0}^{2}\right) k \delta_{2}(k)+4 \xi_{2}^{6}\left(k^{2} b_{0}^{2}\right) k \delta_{2}(k)\left(k^{2} b_{0}^{2}\right) \delta_{2}(k) k+8 \xi_{2}^{4} \xi_{1}^{2}\left(k^{2} b_{0}^{2}\right) k \delta_{2}(k)\left(k^{2} b_{0}^{2}\right) \delta_{2}(k) k+ \\
& 4 \xi_{2}^{2} \xi_{1}^{4}\left(k^{2} b_{0}^{2}\right) k \delta_{2}(k)\left(k^{2} b_{0}^{2}\right) \delta_{2}(k) k-2 \xi_{2}^{4}\left(k^{2} b_{0}^{2}\right) \delta_{1}(k) k b_{0} k \delta_{1}(k)-12 \xi_{2}^{2} \xi_{1}^{2}\left(k^{2} b_{0}^{2}\right) \delta_{1}(k) k b_{0} k \delta_{1}(k)- \\
& 10 \xi_{1}^{4}\left(k^{2} b_{0}^{2}\right) \delta_{1}(k) k b_{0} k \delta_{1}(k)-2 \xi_{2}^{4}\left(k^{2} b_{0}^{2}\right) \delta_{1}(k) k b_{0} \delta_{1}(k) k-8 \xi_{2}^{2} \xi_{1}^{2}\left(k^{2} b_{0}^{2}\right) \delta_{1}(k) k b_{0} \delta_{1}(k) k- \\
& 6 \xi_{1}^{4}\left(k^{2} b_{0}^{2}\right) \delta_{1}(k) k b_{0} \delta_{1}(k) k+4 \xi_{2}^{4} \xi_{1}^{2}\left(k^{2} b_{0}^{2}\right) \delta_{1}(k) k\left(k^{2} b_{0}^{2}\right) k \delta_{1}(k)+8 \xi_{2}^{2} \xi_{1}^{4}\left(k^{2} b_{0}^{2}\right) \delta_{1}(k) k\left(k^{2} b_{0}^{2}\right) k \delta_{1}(k)+ \\
& 4 \xi_{1}^{6}\left(k^{2} b_{0}^{2}\right) \delta_{1}(k) k\left(k^{2} b_{0}^{2}\right) k \delta_{1}(k)+4 \xi_{2}^{4} \xi_{1}^{2}\left(k^{2} b_{0}^{2}\right) \delta_{1}(k) k\left(k^{2} b_{0}^{2}\right) \delta_{1}(k) k+8 \xi_{2}^{2} \xi_{1}^{4}\left(k^{2} b_{0}^{2}\right) \delta_{1}(k) k\left(k^{2} b_{0}^{2}\right) \delta_{1}(k) k+ \\
& 4 \xi_{1}^{6}\left(k^{2} b_{0}^{2}\right) \delta_{1}(k) k\left(k^{2} b_{0}^{2}\right) \delta_{1}(k) k-10 \xi_{2}^{4}\left(k^{2} b_{0}^{2}\right) \delta_{2}(k) k b_{0} k \delta_{2}(k)-12 \xi_{2}^{2} \xi_{1}^{2}\left(k^{2} b_{0}^{2}\right) \delta_{2}(k) k b_{0} k \delta_{2}(k)- \\
& 2 \xi_{1}^{4}\left(k^{2} b_{0}^{2}\right) \delta_{2}(k) k b_{0} k \delta_{2}(k)-6 \xi_{2}^{4}\left(k^{2} b_{0}^{2}\right) \delta_{2}(k) k b_{0} \delta_{2}(k) k-8 \xi_{2}^{2} \xi_{1}^{2}\left(k^{2} b_{0}^{2}\right) \delta_{2}(k) k b_{0} \delta_{2}(k) k- \\
& 2 \xi_{1}^{4}\left(k^{2} b_{0}^{2}\right) \delta_{2}(k) k b_{0} \delta_{2}(k) k+4 \xi_{2}^{6}\left(k^{2} b_{0}^{2}\right) \delta_{2}(k) k\left(k^{2} b_{0}^{2}\right) k \delta_{2}(k)+8 \xi_{2}^{4} \xi_{1}^{2}\left(k^{2} b_{0}^{2}\right) \delta_{2}(k) k\left(k^{2} b_{0}^{2}\right) k \delta_{2}(k)+ \\
& 4 \xi_{2}^{2} \xi_{1}^{4}\left(k^{2} b_{0}^{2}\right) \delta_{2}(k) k\left(k^{2} b_{0}^{2}\right) k \delta_{2}(k)+4 \xi_{2}^{6}\left(k^{2} b_{0}^{2}\right) \delta_{2}(k) k\left(k^{2} b_{0}^{2}\right) \delta_{2}(k) k+8 \xi_{2}^{4} \xi_{1}^{2}\left(k^{2} b_{0}^{2}\right) \delta_{2}(k) k\left(k^{2} b_{0}^{2}\right) \delta_{2}(k) k+ \\
& 4 \xi_{2}^{2} \xi_{1}^{4}\left(k^{2} b_{0}^{2}\right) \delta_{2}(k) k\left(k^{2} b_{0}^{2}\right) \delta_{2}(k) k+8 \xi_{2}^{4} \xi_{1}^{2}\left(k^{4} b_{0}^{3}\right) k \delta_{1}(k) b_{0} k \delta_{1}(k)+16 \xi_{2}^{2} \xi_{1}^{4}\left(k^{4} b_{0}^{3}\right) k \delta_{1}(k) b_{0} k \delta_{1}(k)+
\end{aligned}
$$


$8 \xi_{1}^{6}\left(k^{4} b_{0}^{3}\right) k \delta_{1}(k) b_{0} k \delta_{1}(k)+8 \xi_{2}^{4} \xi_{1}^{2}\left(k^{4} b_{0}^{3}\right) k \delta_{1}(k) b_{0} \delta_{1}(k) k+16 \xi_{2}^{2} \xi_{1}^{4}\left(k^{4} b_{0}^{3}\right) k \delta_{1}(k) b_{0} \delta_{1}(k) k+$ $8 \xi_{1}^{6}\left(k^{4} b_{0}^{3}\right) k \delta_{1}(k) b_{0} \delta_{1}(k) k+8 \xi_{2}^{6}\left(k^{4} b_{0}^{3}\right) k \delta_{2}(k) b_{0} k \delta_{2}(k)+16 \xi_{2}^{4} \xi_{1}^{2}\left(k^{4} b_{0}^{3}\right) k \delta_{2}(k) b_{0} k \delta_{2}(k)+$ $8 \xi_{2}^{2} \xi_{1}^{4}\left(k^{4} b_{0}^{3}\right) k \delta_{2}(k) b_{0} k \delta_{2}(k)+8 \xi_{2}^{6}\left(k^{4} b_{0}^{3}\right) k \delta_{2}(k) b_{0} \delta_{2}(k) k+16 \xi_{2}^{4} \xi_{1}^{2}\left(k^{4} b_{0}^{3}\right) k \delta_{2}(k) b_{0} \delta_{2}(k) k+$ $8 \xi_{2}^{2} \xi_{1}^{4}\left(k^{4} b_{0}^{3}\right) k \delta_{2}(k) b_{0} \delta_{2}(k) k+8 \xi_{2}^{4} \xi_{1}^{2}\left(k^{4} b_{0}^{3}\right) \delta_{1}(k) k b_{0} k \delta_{1}(k)+16 \xi_{2}^{2} \xi_{1}^{4}\left(k^{4} b_{0}^{3}\right) \delta_{1}(k) k b_{0} k \delta_{1}(k)+$ $8 \xi_{1}^{6}\left(k^{4} b_{0}^{3}\right) \delta_{1}(k) k b_{0} k \delta_{1}(k)+8 \xi_{2}^{4} \xi_{1}^{2}\left(k^{4} b_{0}^{3}\right) \delta_{1}(k) k b_{0} \delta_{1}(k) k+16 \xi_{2}^{2} \xi_{1}^{4}\left(k^{4} b_{0}^{3}\right) \delta_{1}(k) k b_{0} \delta_{1}(k) k+$ $8 \xi_{1}^{6}\left(k^{4} b_{0}^{3}\right) \delta_{1}(k) k b_{0} \delta_{1}(k) k+8 \xi_{2}^{6}\left(k^{4} b_{0}^{3}\right) \delta_{2}(k) k b_{0} k \delta_{2}(k)+16 \xi_{2}^{4} \xi_{1}^{2}\left(k^{4} b_{0}^{3}\right) \delta_{2}(k) k b_{0} k \delta_{2}(k)+$ $8 \xi_{2}^{2} \xi_{1}^{4}\left(k^{4} b_{0}^{3}\right) \delta_{2}(k) k b_{0} k \delta_{2}(k)+8 \xi_{2}^{6}\left(k^{4} b_{0}^{3}\right) \delta_{2}(k) k b_{0} \delta_{2}(k) k+16 \xi_{2}^{4} \xi_{1}^{2}\left(k^{4} b_{0}^{3}\right) \delta_{2}(k) k b_{0} \delta_{2}(k) k+$ $8 \xi_{2}^{2} \xi_{1}^{4}\left(k^{4} b_{0}^{3}\right) \delta_{2}(k) k b_{0} \delta_{2}(k) k$.

\section{REFERENCES}

[1] Michael T. Anderson, Geometrization of 3-manifolds via the Ricci flow. Notices Amer. Math. Soc. 51 (2004), no. 2, 184-193.

[2] Michael T. Anderson, Singularities of the Ricci flow, in Encyclopedia of Mathematical Physics, pp.584-588, Elsevier 2006.

[3] M. Bañados, C. Teitelboim, J. Zanelli, Black hole in three-dimensional spacetime. Phys. Rev. Lett. 69 (1992), no. 13, 1849-1851.

[4] Shigetoshi Bando and Hajime Urakawa, Generic properties of the eigenvalue of the Laplacian for compact Riemannian manifolds, Tohoku Math. J. (2) 35 (1983), no. 2, 155-172.

[5] P. Bieliavsky, S. Detournay, M. Rooman, Ph. Spindel, BTZ black holes, WZW models and noncommutative geometry, arXiv:hep-th/0511080

[6] Marcel Berger, Sur les premières valeurs propres des varités riemanniennes, Compositio Math. 26 (1973), 129-149.

[7] D. Birmingham, C. Kennedy, S. Sen, and A. Wilkins, Geometrical finiteness, holography, and the Bañados-Teitelboim-Zanelli black hole. Phys. Rev. Lett. 82 (1999), N.21, 4164-4167.

[8] Xiaodong Cao, Eigenvalues of $-\Delta+R / 2$ on manifolds with nonnegative curvature operator, Math. Ann. 337 (2007), no. 2, 435-441.

[9] Xiaodong Cao, First eigenvalues of geometric operators under the Ricci flow, Proc. Amer. Math. Soc., Vol.136 (2008) N.11, 4075-4078.

[10] H.D. Cao, X.P. Zhu, Hamilton-Perelman's Proof of the Poincaré Conjecture and the Geometrization Conjecture, arXiv:math/0612069.

[11] Mauro Carfora, Renormalization group and the Ricci flow, arXiv:1001.3595

[12] Ali H. Chamseddine and Alain Connes, The spectral action principle, Comm. Math. Phys. 186 (1997), no. 3, 731-750.

[13] Bennett Chow, The Ricci flow on the 2-sphere. J. Differential Geometry, Vol.33 (1991) 325334.

[14] Bennett Chow, Peng Lu, Lei Ni, Hamilton's Ricci flow, American Mathematical Society, 2006.

[15] Alain Connes, $C^{*}$ algèbres et géométrie différentielle, C. R. Acad. Sci. Paris Sér. A-B 290 (1980), no. 13, A599-A604.

[16] Alain Connes, Noncommutative geometry, Academic Press, 1994.

[17] Alain Connes, Geometry from the spectral point of view. Lett. Math. Phys. 34 (1995), no. 3, 203238.

[18] Alain Connes and Joachim Cuntz, Quasi homomorphismes, cohomologie cyclique e positivité. Comm. Math. Phys., Vol.11 (1988) N.3, 515-526.

[19] Alain Connes and Michel Dubois-Violette, Moduli space and structure of non commutative 3-spheres, Lett. Math. Phys. 66 (2003), no. 1-2, 91-121.

[20] Alain Connes and Paula Tretkoff, The Gauss-Bonet theorem for the noncommutative two torus. arXiv:math/0910.0188v1 [math.QA] 1 Oct 2009.

[21] Gunther Cornelissen and Jan Willem de Jong, The spectral length of a map between Riemannian manifolds, arXiv:1007.0907

[22] Gunther Cornelissen and Matilde Marcolli, Zeta functions that hear the shape of a Riemann surface, Journal of Geometry and Physics, Vol.58 (2008) N.5, 619-632

[23] Gunther Cornelissen and Matilde Marcolli, Quantum statistical mechanics, L-series and anabelian geometry, arXiv:1009.0736. 
[24] Xianzhe Dai and Li Ma, Mass under the Ricci flow, Commun. Math. Phys., 274 (2007) N.1, 65-80.

[25] Luca Fabrizio Di Cerbo, Eigenvalues of the Laplacian under the Ricci flow. Rendiconti di Matematica, Roma, Serie VII, Volume 27 (2007) 183-195.

[26] B.P. Dolan, K.S. Gupta, A. Stern, Noncommutative BTZ black hole and discrete time, Classical Quantum Gravity 24 (2007), no. 6, 1647-1655.

[27] C.Y. Ee, D. Lee, Y. Lee, The noncommutative BTZ black hole in polar coordinates, Classical Quantum Gravity 26 (2009), no. 18, 185001, 10 pp.

[28] D. Friedan Nonlinear models in two + epsilon dimensions, Phys. Rev. Lett. 45 (1980) 1057

[29] Farzad Fathi-Zadeh and Masoud Khalkhali, The Gauss-Bonnet theorem for noncommutative two tori with a general conformal structure, arXiv:math/1005.4947v1 [math.OA] 26 May 2010.

[30] Peter B. Gilkey, Invariance theory, the heat equation, and the Atiyah-Singer index theorem, Second Edition, CRC Press, 1995.

[31] Matthew Headrick and Toby Wiseman, Ricci flow and black holes, Classical Quantum Gravity 23 (2006) N.23, 6683-6707.

[32] Richard S. Hamilton, Three-manifolds with positive Ricci curvature, J. Differential Geometry, Vol.17 (1982) N.2, 255-306.

[33] Richard S. Hamilton, The Ricci flow on surfaces, in "Mathematics and general relativity (Santa Cruz, CA, 1986)", Contemp. Math., Vol.71 (1988) 237-262, Amer. Math. Soc., Providence, RI, 1988.

[34] Richard S. Hamilton, An isoperimetric estimate for the Ricci flow on the two-sphere, in "Modern methods in complex analysis (Princeton, NJ, 1992)", 191-200, Ann. of Math. Stud., 137, Princeton Univ. Press, Princeton, NJ, 1995.

[35] Richard S. Hamilton, The Harnack estimate for the Ricci flow, J. Differential Geom. 37 (1993), no. 1, 225-243.

[36] Richard S. Hamilton, Eternal solutions to the Ricci flow, J. Differential Geom. 38 (1993), no. $1,1-11$.

[37] Richard S. Hamilton, The formation of singularities in the Ricci flow. Surveys in differential geometry, Vol. II (Cambridge, MA, 1993), 7-136, Int. Press, Cambridge, MA, 1995.

[38] Richard S. Hamilton and Shing-Tung Yau, The Harnack estimate for the Ricci flow on a surface - revisited, Asian J. Math. 1 (1997), no. 3, 418-421.

[39] Kirill Krasnov, Holography and Riemann surfaces, Adv. Theor. Math. Phys. 4 (2000), no. 4, 929-979.

[40] A. Kokotov, D. Korotkin, Normalized Ricci flow on Riemann surfaces and determinant of Laplacian, Lett. Math. Phys. 71 (2005), no. 3, 241-242.

[41] Jun-Fang Li, Eigenvalues and energy functionals with monotonicity formulae under Ricci flow, Math. Ann. 338 (2007), no. 4, 927-946.

[42] B. Kleiner, J. Lott, Notes on Perelman's papers. Geom. Topol. 12 (2008), no. 5, 2587-2855.

[43] Li Ma, Eigenvalue monotonicity for the Ricci-Hamilton flow, Ann. Global Anal. Geom. 29 (2006), no. 3, 287-292.

[44] Sylvain Maillot, Ricci flow, scalar curvature and the Poincaré Conjecture, in "Geometry, Topology, Quantum Field Theory and Cosmology" Physique - Mathématiques Travaux en Cours, pp.97-116, Hermann Éditeurs, 2009.

[45] Yuri I. Manin and Matilde Marcolli, Holography principle and arithmetic of algebraic curves. Adv. Theor. Math. Phys. 5 (2001), no. 3, 617-650.

[46] Varghese Mathai and Jonathan Rosenberg, A noncommutative sigma-model, Journal of Noncommutative Geometry, vol. 5 (2011) no.2, 265-294.

[47] John Morgan and Gang Tian, Ricci flow and the Poincaré conjecture, Clay Mathematics Monographs, 3. American Mathematical Society, Providence, RI; Clay Mathematics Institute, Cambridge, MA, 2007. xlii+521 pp.

[48] B. Osgood, R. Phillips, P. Sarnak, Extremals of determinants of Laplacians, J. Funct. Anal. 80 (1988), 148-211.

[49] Grisha Perelman, The entropy formula for the Ricci flow and its geometric applications. arXiv:math/0211159v1 [math.DG] 11 Nov 2002.

[50] Grisha Perelman, Ricci flow with surgery on three-manifolds. arXiv:math/0303109v1 [math.DG] 10 Mar 2003. 
[51] Grisha Perelman, Finite extinction time for the solutions to the Ricci flow on certain threemanifolds. arXiv:math/0307245v1 [math.DG] 17 Jul 2003.

[52] Steven Rosenberg, The Laplacian on a Riemannian manifold. Cambridge University Press, 1997.

[53] Simon Scott, Traces and determinants of pseudodifferential operators, Oxford University Press, 2010.

[54] Sergiu I. Vacaru, Spectral functionals, nonholonomic Dirac operators, and noncommutative Ricci flows, J. Math. Phys. 50, 073503 (2009) [24 pages]

[55] Joseph C. Varilly, An introduction to noncommutative geometry. European Mathematical Society, 2006.

Department of Mathematics, California Institute of Technology, Pasadena, CA 91125, USA

E-mail address: tanvirab@caltech.edu

E-mail address: matilde@caltech.edu 